\title{
Lens correction algorithm based on the see-saw diagram to correct Seidel aberrations employing aspheric surfaces
}

\author{
Martha Rosete-Aguilar
}

Martha Rosete-Aguilar, "Lens correction algorithm based on the see-saw diagram to correct Seidel aberrations employing aspheric surfaces," Proc. SPIE 3831, Sixth International Conference on Education and Training in Optics and Photonics, (16 June 2000); doi: 10.1117/12.388707 


\title{
A lens correction algorithm based on the see-saw diagram to correct Seidel aberrations employing aspheric surfaces
}

\author{
Martha Rosete-Aguilar \\ Laboratorio de Optica Aplicada, Centro de Instrumentos, U.N.A.M. Circuito Exterior, \\ Cd. Universitaria, México, D.F., 04510, México.
}

\begin{abstract}
In this paper a lens correction algorithm based on the see-saw diagram developed by Burch [1] is described. The see-saw diagram describes the image correction in rotationally symmetric systems over a finite field of view by means of aspherics surfaces. The algorithm is applied to the design of some basic telescopic configurations such as the classical Cassegrain telescope, the Dall-Kirkham telescope, the Pressman-Camichel telescope and the Ritchey-Chretien telescope in order to show a physically visualizable concept of image correction for optical systems that employ aspheric surfaces. By using the see-saw method the student can visualise the different possible configurations of such telescopes as well as their performances and also the student will be able to understand that it is not always possible to correct more primary aberrations by aspherizing more surfaces.
\end{abstract}

Keywords: Seidel aberrations, aspherics, aberrations, see-saw method, telescopes.

\section{INTRODUCTION}

In this paper a lens correction algorithm based on the see-saw diagram developed by Burch is described and applied to the design of some basic telescopic configurations in order to show a physical concept that enables us to understand how image correction may be achieved when using aspheric surfaces. The see-saw diagram describes the image correction in rotationally symmetric systems and it determines what aspheric shape and position the surfaces should have in order to obtain good performance over a finite field of view. The see-saw method neglects higher order terms in the wavefront aberration expansion, i.e.. aberrations higher than Seidel's. In other words only the Seidel aberrations, primary spherical aberration, coma. astigmatism and distortion may be corrected when using this method. One question may arise: If we already know how to evaluate the Seidel aberration terms then what is the use about of the see-saw method? The answer was given by Burch [2] and is that because it uses physically visualizable concepts, it enables us to think physically, as well as mathematically. A physical picture of the problem may suggest more solutions and a good example of this statement is the telescope designed by Gascoigne[3]. Gascoigne achieved a previously overlooked design of a Schmidt-Cassegrain telescope with the corrector plate located in the converging beam in the image space by using the see-saw method.

\section{THE SEE-SAW METHOD CONCEPT}

The idea of the see-saw method is based on the fact that a spherical surface with an aspheric corrector plate located at its centre of curvature is corrected for the primary aberrations, spherical aberration, coma and astigmatism. The corrector plate is aspherized to correct the primary spherical aberration of the spherical surface. This optical system -spherical surface + aspheric corrector plate- is called anastigmatic which means that the optical system produces primary spherical image wavefronts, that is, the Seidel coefficients for spherical aberration, coma and astigmatism are all zero. This is the principle of the Schmidt telescope and the reason why this telescope has a good performance over a wide field of view on a spherical surface.

Now, if any spherical surface can be anastigmatized by locating an aspheric corrector plate at its centre of curvature thus any optical system composed of a set of rotationally symmetric spherical surfaces can be anastigmatized if each surface is anastigmatized with an aspheric corrector plate (also called anastigmatizing corrector plate).

A practical problem arises when the surface and its corresponding corrector plate are intercepted by any other surface of the system or if the corrector plate must be located in a non-physical real space as happens with a convex spherical mirror with its centre of curvature in a virtual space. This can be overcome, however, by imaging the corrector plate into a "star space". Star-space means the space where the object is at infinity, among the stars. If no star-space exists in the system as given, it is 
permissible to add a hypothetical anastigmat of any convenient focal length to create a star-space into which the corrector plate may be imaged.

But before discussing an optical system composed of several spherical surfaces, let us consider the simplest case which is a system consisting of only one spherical surface.

\section{WAVEFRONT ABERRATION AT A SPHERICAL SURFACE FOR AN AXIAL OBJECT POINT}

Let $n, n^{\prime}$ be the refractive indices of the media before and after refraction (or reflection in which case $n^{\prime}=-n$ ), respectively. Let $\rho$ be the paraxial radius of curvature of the surface and $Y_{S}$ be the height at which the wavefront deformation is to be evaluated, see figure 1. For an object on axis, $\mathrm{O}$, the spherical surface produces wavefronts with spherical aberration. To third order approximation, it produces a primary spherical wavefront aberration which varies proportional to the fourth power of the height $Y_{S}$, that is,

$$
W_{S}=\frac{1}{8}\left(\frac{n}{n^{\prime}}\right)^{2}\left(\frac{1}{\rho^{3}}\right)\left(n-n^{\prime}\right)\left(\frac{l-\rho}{l}\right)^{2}\left[\frac{\left(n^{\prime}+n\right) \rho-n l}{n l}\right] Y_{S}^{4}
$$

where $l$ is the distance between the axial object point $O$ located in $n$-space and the vertex of the spherical surface.

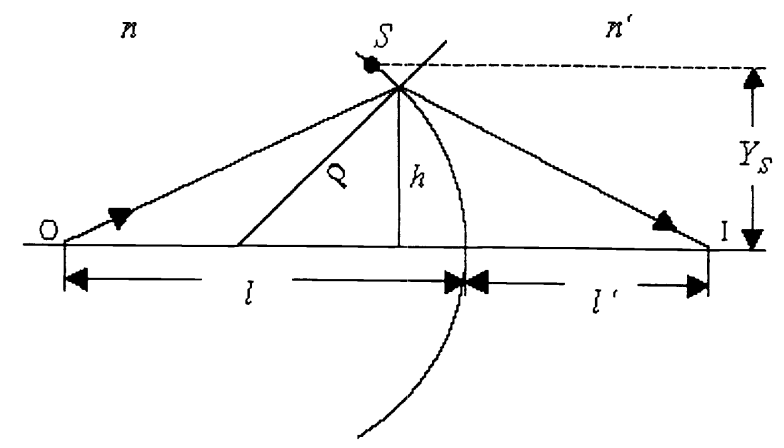

Figure 1. Wavefront aberration at a spherical surface for an axial object point.

\section{RETARDATION REQUIRED BY THE CORRECTOR PLATE}

In order to correct the primary spherical wavefront aberration produced by the spherical surface and given by equation (1), a corrector plate is used. One face of the plate is plane and the other face is aspherized to compensate for the spherical aberration. A first thought could be that this system is only corrected for spherical aberration and this is true if the corrector plate is located at any position which is not the centre of curvature of the surface. One of the great achievements by Schmidt, however, was to realize that by placing the corrector plate at the centre of curvature of the surface not only primary spherical aberration could be corrected but also the primary aberrations: coma and astigmatism.

Now, if the corrector plate is located at the centre of curvature of the surface, the question is: What is the retardation required by the corrector plate to correct the primary spherical wavefront aberration produced by the surface and given by equation (1)? The answer to this question is that there are two different expressions depending on whether the corrector plate is located in $n$-space or $n^{\prime}$-space, in other words it depends on whether the centre of curvature of the spherical surface is to the left or to the right of its vertex. In reference to figures (2.a) and (2.b) let us consider two cases:

Case A: If the corrector plate is located in $n$-space, the paraxial ray, coming from $\mathrm{O}$ and hitting the spherical surface at $\mathrm{S}$, hits the corrector plate at height $Y_{S P}$, see figure (2.a):

$$
Y_{S P}=\left(\frac{l}{l-\rho}\right) Y_{S}
$$

so that the corrector plate should produce a retardation in the wave front given by

$$
W_{S P_{n}}=-\frac{1}{8}\left(\frac{n}{n^{\prime}}\right)^{2}\left(\frac{1}{\rho^{3}}\right)\left(n-n^{\prime}\right)\left(\frac{l}{l-\rho}\right)^{2}\left[\frac{\left(n^{\prime}+n\right) \rho-n l}{n l}\right] Y_{S P}^{4}
$$




$$
W_{S P_{n}}=\tau_{n} Y_{S P}^{4}
$$

where $\rho$ is taken as negative.

Case B: If the corrector plate is located in $n^{\prime}$-space, the paraxial ray, coming from axial object point $\mathrm{O}$, hits the corrector plate at height $Y_{S P}$, see figure (2.b):

$$
Y_{S P}=\left(\frac{n}{n^{\prime}}\right)\left(\frac{l}{\rho-l}\right) Y_{S}
$$

so that the corrector plate should produce a retardation in the wave front given by

$$
\begin{gathered}
W_{S P_{n^{\prime}}}=-\frac{1}{8}\left(\frac{n^{\prime}}{n}\right)^{2}\left(\frac{1}{\rho^{3}}\right)\left(n-n^{\prime}\right)\left(\frac{l}{l-\rho}\right)^{2}\left[\frac{\left(n^{\prime}+n\right) \rho-n l}{n l}\right] Y_{S P}^{4} \\
W_{S P_{n^{\prime}}}=\tau_{n^{\prime}} Y_{S P}^{4}
\end{gathered}
$$$$
\text { or }
$$

where $\rho$ is taken as positive.

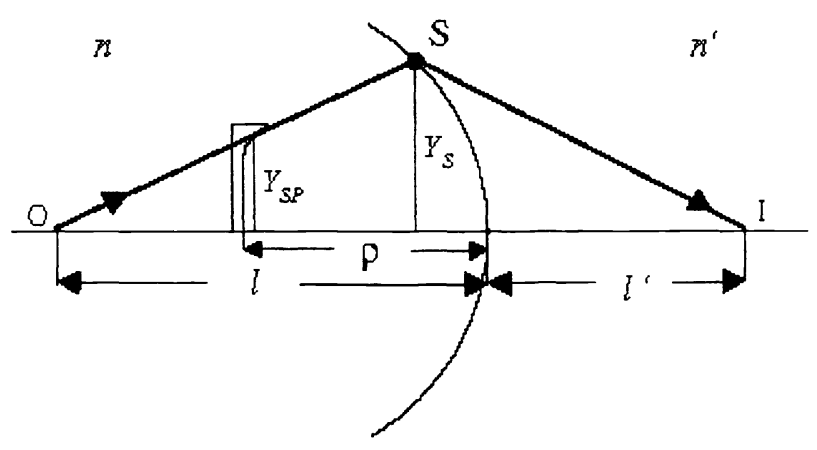

(a)

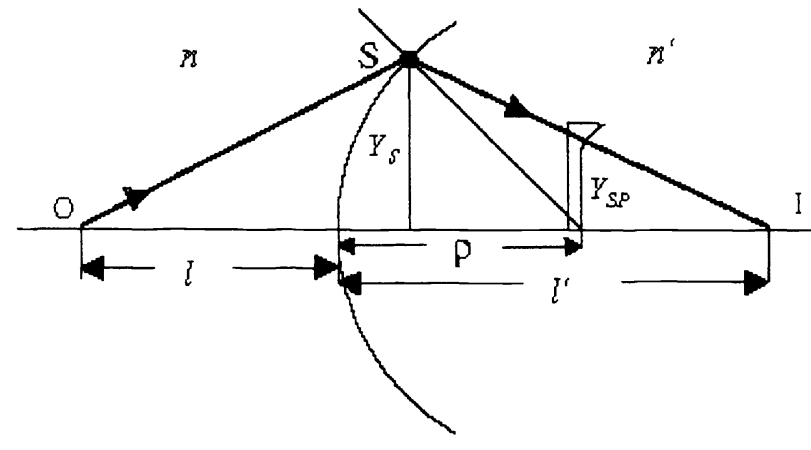

(b)

Figure 2. Retardation required by the corrector plate when the corrector is located in (a) $n$-space and (b) $n$ '-space.

\section{STRENGTH OF A CORRECTOR PLATE}

The retardation that the corrector plate should produce to the wavefront to compensate for the primary spherical aberration of the spherical surface is given by Eq. (3) or Eq. (6). In an optical system, however, the corrector plates are ideal components that are not present in the system. For this reason, we may called these plates as missing corrector plates. The wavefront aberration at the missing corrector plates is the negative of the wavefront given by Eq. (3) for case A or Eq. (6) for case B. That is,

$$
W_{S P_{j}}=-\tau_{j} Y_{S P}^{4}=\mu_{j} Y_{S P}^{4}
$$

where $\mu_{j}$ (with $j=n$ or $n^{\prime}$ ) is called the strength of the missing corrector plate.

In other words, the strength of the missing corrector plate is the wavefront aberration produce by the spherical surface at its centre of curvature.

\section{THE SEE-SAW DIAGRAM}

Let us now assume that our optical system has more than one surface. We evaluate the strength, $\mu_{j}$, of each missing corrector plate and then all plates are imaged into star-space by that part of the system that precedes them, according to Gaussian optics. When doing this, the strength of each missing corrector plate, $\mu$, is altered by the magnification of the missing corrector plate, $M$, when is imaged into star-space. The new strength of the plate in star-space is given by,

$$
\alpha_{\substack{\text { star } \\ \text { space }}}=\frac{\mu}{M^{4}}
$$


The relative position between the missing corrector plates also changes after being imaged into star-space. Now, forget the system, and simply draw the diagram of the plates with strengths given by $\alpha_{i}$ and their relative positions in star-space given by $x_{i}$. This is the see-saw diagram, see figure 3 . Up to now we have not considered the case when any of the surfaces of the system is already aspheric or if one or more of the surfaces is going to be aspherized. In this case, the strength of the surfaces is given by Eq. (1), i.e., the wavefront aberration at the surface is given by: $W_{S}=\eta Y_{S}^{4}$ where $\eta$ is the strength of the surface. In the same way as with the missing corrector plates, the surfaces have to be imaged into star-space taking into account the magnification which will affect the strength $\eta$ as:

$$
\gamma_{\text {star }}=\frac{\eta}{M^{4}}
$$

In figure 3, we have included the strenght of these surfaces in star space as $\gamma_{1}$ and $\gamma_{2}$.

Let $\alpha_{1}, \alpha_{2}, \alpha_{3}, \ldots, \alpha_{n}$ be the strength of the missing corrector plates and let $x_{1}, x_{2}, x_{3}, \ldots, x_{n}$ be their respective distances measured with respect to an origin, PP, that corresponds to the position of the entrance pupil of the system. Let $\gamma_{1}, \gamma_{2}, \gamma_{3}, \ldots, \gamma_{n}$ be the strength of the surfaces (if the surface is spherical then $\gamma=0$ ) and let $v_{1}, v_{2}, v_{3}, \ldots, v_{n}$ be their respective distances measured with respect to PP. The distances are measured from the pivot point, PP, negative to the left of PP and positive to the right of PP. If all these plates are considered to be masses proportional to their strength, the first three aberrations in the optical system, spherical aberration, coma and astigmatism are the analogy of forces applied to a physical system, that is,

Spherical aberration is given by the total weight on the pivot point:

$$
m_{1}=\sum_{i=1}^{n} \alpha_{i}+\sum_{i=1}^{n} \gamma_{i}
$$

Coma is proportional to the unbalanced moment about the pivot point:

$$
m_{2}=\sum_{i=1}^{n} \alpha_{i} x_{i}+\sum_{i=1}^{n} \gamma_{i} \nu_{i}
$$

Astigmatism is proportional to the moment of inertia about the pivot point:

$$
m_{3}=\sum_{i=1}^{n} \alpha_{i} x_{i}^{2}+\sum_{i=1}^{n} \gamma_{i} v_{i}^{2}
$$

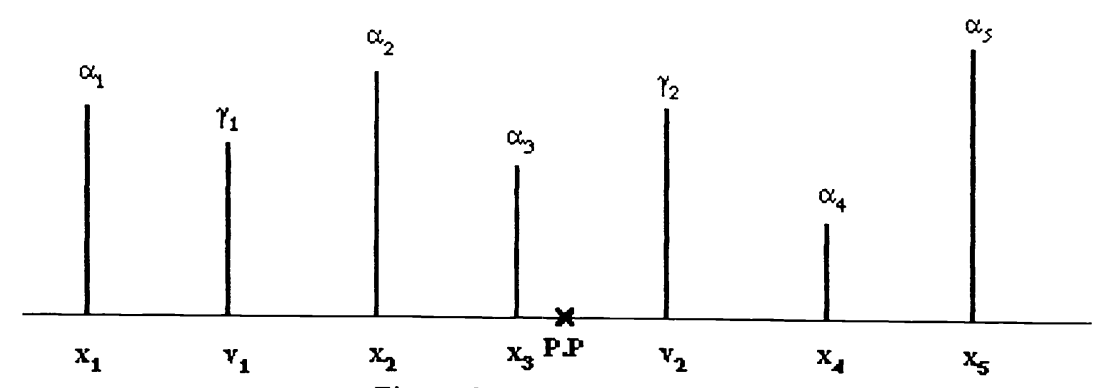

Figure 3. See-saw diagram.

The terms $m_{1}, m_{2}, m_{3}$ are the zeroth, first and second moments of the see-saw diagram, respectively. In the context of optical design, if the optical system is in equilibrium, all moments are annulled and therefore the system is anastigmatic. The expression for distortion is not given here since the method will be applied to the design of telescopes where distortion may be neglected in the design.

\section{LENS CORRECTION ALGORITHM BASED ON THE SEE-SAW DIAGRAM}

The algorithm that we have written is able to construct the see-saw diagram as shown in figure 3. To do this, it is necessary to evaluate the position of both the missing corrector plates and the surfaces in the optical system in star-space. The position of each missing corrector plate (or a surface) is determined by imaging it through that part of the system that precedes it according to Gaussian optics. In the process of imaging the missing corrector plate (or the surface), its magnification, $M$, is evaluated. It is also necessary to evaluate the strength of the missing corrector plates (or the surfaces). The strength, $\mu$, of 
the missing corrector plate is evaluated through the negative of Eqs. (3) or (6) (or Eq. (1) for the strength $\eta$ of a surface)). And then, the strengh in star space, $\alpha$, is evaluated through Eq. (9), (or the strength $\gamma$ for a surface is evaluated through Eq. (10)). After evaluating both strengths and positions of the missing corrector plates and surfaces, the see-saw diagram can be drawn. We can now write a set of equations given by Eqs.(11), (12) and (13). To solve one, two or three of these equations will depend on how many aberrations we want to correct.

\section{APPLICATION OF THE SEE-SAW METHOD TO THE DESIGN OF CASSEGRAIN TYPE}

\section{TELESCOPES}

A basic configuration of a two mirror telescope with a primary concave mirror PM and a secondary convex mirror SM is presented in this section. Let $R_{1}, R_{2}$ be the radii of curvature of the primary concave mirror and the secondary convex mirror respectively, both being negative and let $d$ (taken as negative) be the separation between the mirrors. The stop is placed at the primary mirror PM.

Let $\alpha_{1}$ and $\alpha_{2}$ be the strength of the missing corrector plates corresponding to the primary mirror and the secondary mirror respectively. Let $\gamma_{1}$ be the strength of the primary mirror and let $\gamma_{2}$ be the strength of the secondary mirror, where $\gamma_{1}$ and $\gamma_{2}$ are equal to zero if the mirrors are spherical. Since the stop is at the primary mirror which is already in starspace, the origin is set at the primary mirror from where all other distances will be measured. The position, $v_{1}$, and the magnification, $M_{1}$, for the primary mirror are given by,

$$
\begin{aligned}
& v_{1}=0 \\
& M_{1}=1
\end{aligned}
$$

The secondary mirror is imaged into star-space, where its position, $v_{2}$, with respect to the primary mirror and its magnification, $M_{2}$, are given according to Gaussian optics, by

$$
\begin{aligned}
& v_{2}=\frac{R_{1} d}{2 d-R_{1}} \\
& M_{2}=\frac{R_{1}}{R_{1}-2 d}
\end{aligned}
$$

The corrector plate corresponding to the primary mirror is already in star-space, so its position and magnification in starspace are,

$$
\begin{aligned}
& x_{1}=R_{1} \\
& M_{1}=1
\end{aligned}
$$

whereas the strength of the corrector plate corresponding to the primary mirror, $\alpha_{1}$, is given by,

$$
\alpha_{1}=-\frac{1}{4}\left(\frac{1}{R_{1}^{3}}\right) \text {. }
$$

The corrector plate corresponding to the secondary mirror is imaged into star-space, so its position $x_{2}$, and its magnification, $M_{2}$ in star-space are given by,

$$
\begin{aligned}
& x_{2}=\frac{R_{1}\left(d+R_{2}\right)}{2 d+2 R_{2}-R_{1}} \\
& M_{2}=\frac{R_{1}}{R_{1}-2 d-2 R_{2}}
\end{aligned}
$$

whereas the strength of the corrector plate, corresponding to the secondary mirror, in star-space is,

$$
\alpha_{2}=\frac{1}{4}\left(\frac{1}{R_{2}^{3}}\right)\left(\frac{1}{R_{1}^{4}}\right)\left(R_{1}-2 d\right)^{2}\left(R_{1}-2 d-2 R_{2}\right)^{2} \text {. }
$$


The see-saw diagram for this system is shown in figure 4, where the distances are negative to the left of the pivot-point or entrance pupil, PP.

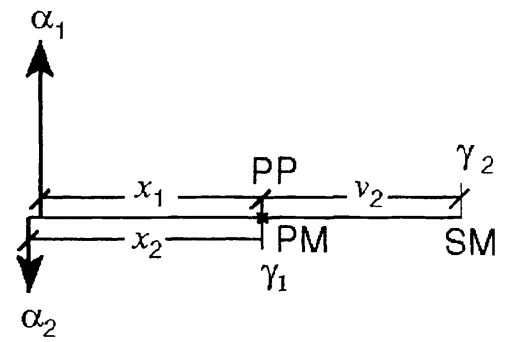

Figure 4. See-saw diagram for a two mirror system.

The total spherical aberration of this system is given by,

$$
\alpha_{1}+\alpha_{2}+\gamma_{1}+\gamma_{2}
$$

and the proportional value of coma is given by,

$$
\alpha_{1} x_{1}+\alpha_{2} x_{2}+\gamma_{1} v_{1}+\gamma_{2} v_{2}
$$

where $x_{1}, x_{2}$ are the positions in star space of the corrector plates and $v_{1}, v_{2}$ are the positions in star space of the primary and secondary mirrors respectively.

As we can see, a two mirror system has a plate diagram containing four plates, and for a given mirror pair and spacing not only the strength of two of these plates are fixed but also the spacing between the four plates. In other words, we can always choose the figurings of the mirrors for correction of primary spherical aberration and coma but we can not, in general, make the astigmatism zero.

\subsection{Design of a classical-Cassegrain telescope}

The see-saw diagram for the classical-Cassegrain telescope is shown in figure 5(a). The primary mirror PM in a classicalCassegrain is parabolic so the image at the prime focus is free from spherical aberration. The strength of the aspheric profile on the primary mirror is, therefore, equal to the strength of the anastigmatizing corrector plate, i.e., $\gamma_{1}=-\alpha_{1}$. To correct the total spherical aberration of the system given by Eq. (24), the secondary mirror SM needs a strength equal to $\gamma_{2}=-\alpha_{2}$. Coma is proportional to the unbalanced momentum given by Eq. (25). In figure 5(b) an schematic diagram shows the evaluation of coma by means of vectors that show the magnitude for each term of the unbalanced moment given by $\gamma_{2} v_{2}+\sum_{i=1}^{2} \alpha_{i} x_{i}$.

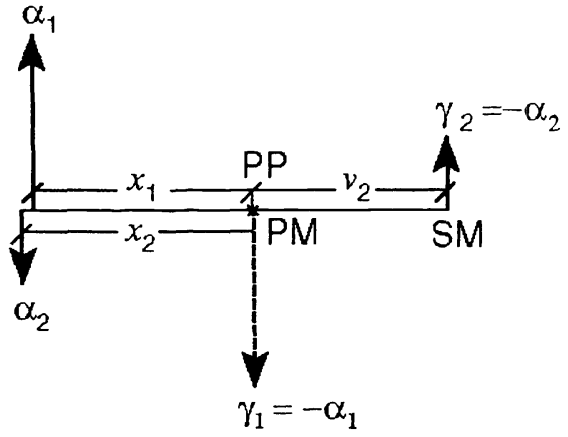

(a)

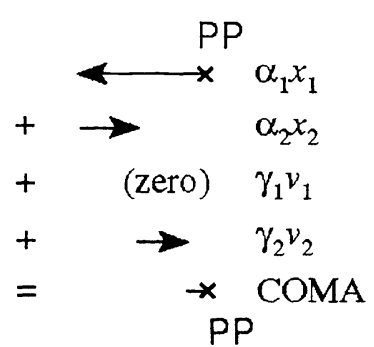

(b)

Figure 5. (a) See-saw diagram for a Classical-Cassegrain telescope and (b) schematic diagram for evaluation of coma. 


\subsection{Design of a Dall-Kirkham telescope}

The see-saw diagram for the Dall-Kirkham telescope is shown in figure 6(a). In this telescope the secondary mirror is spherical, i.e., $\gamma_{2}=0$, so that the spherical aberration of the system has to be corrected by the primary mirror alone, that is, $\gamma_{1}=-\left(\alpha_{1}+\alpha_{2}\right)$

Coma is proportional to the unbalanced moment given by $\sum_{i=1}^{2} \alpha_{i} x_{i}$ as shown in figure 6(b).

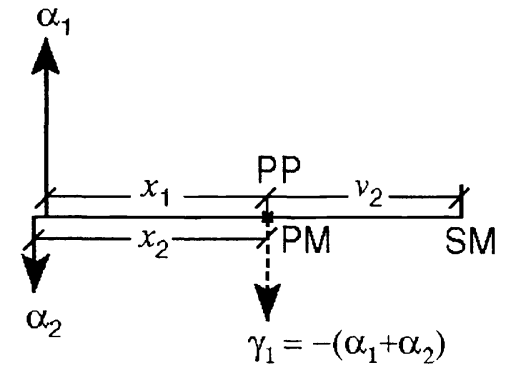

(a)

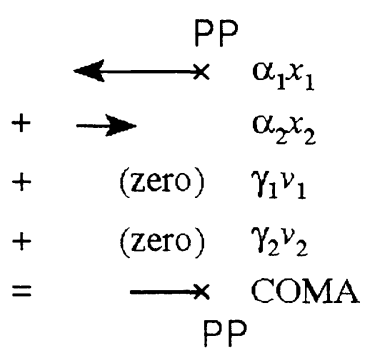

(b)

Figure 6. (a) See-saw diagram for a Dall-Kirkham telescope and (b) schematic diagram for evaluation of coma.

\subsection{Design of a Pressman-Camichel telescope}

The see-saw diagram for the Pressman-Camichel telescope is presented in figure 7(a). In this telescope the primary mirror is spherical. The secondary mirror is aspherized to correct the spherical aberration of the system, its strength should then be equal to $\gamma_{2}=-\left(\alpha_{1}+\alpha_{2}\right)$. In this telescope, coma is proportional to, $-\left(\alpha_{1}+\alpha_{2}\right) v_{2}+\sum_{i=1}^{2} \alpha_{i} x_{i}$.

As in the previous designs, a schematic representation with vectors on figure 7(b) shows the proportional value of coma which is the aberration that limits the field of view in all these telescopes. By comparing Figs. 5(b), 6(b) and 7(b) we can see that the coma for the classical Cassegrain telescope is smaller than for the other two telescopes.

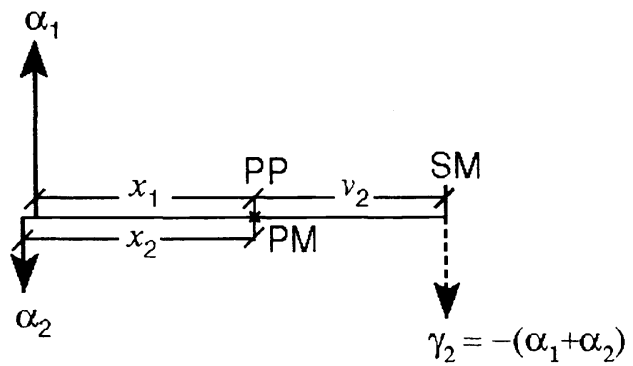

(a)

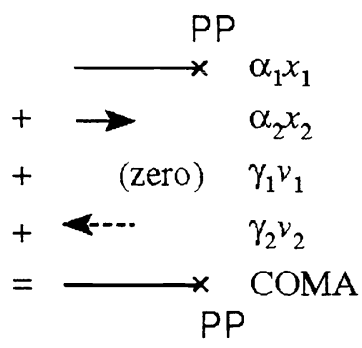

(b)

Figure 7. (a) See-saw diagram for a Pressman-Camichel telescope and (b) schematic diagram for evaluation of coma.

\subsection{Design of a Ritchey-Chretien telescope}

The last design in this type of configuration is the Ritchey-Chretien telescope. In this telescope neither the primary nor the secondary mirrors are aspherized to a particular shape as in the previous examples. On the contrary, the purpose of this telescope is to correct for the spherical aberration and coma, therefore the shape of both mirrors are such that they balance the zeroth and first moments given by the missing corrector plates. In other words, the strength of both mirrors are chosen so that,

$$
\text { and } \begin{gathered}
\alpha_{1}+\alpha_{2}+\gamma_{1}+\gamma_{2}=0 \\
\gamma_{2} v_{2}+\alpha_{1} x_{1}+\alpha_{2} x_{2}=0
\end{gathered}
$$

The see-saw diagram for this telescope is shown in figure 8 (a) and a schematic diagram with vectors showing the evaluation of coma is shown in figure $8(\mathrm{~b})$. In this telescope the field of view is limited by astigmatism. 


\section{References}

1. C.R. Burch, "On the optical see-saw diagram", Mon. Not. R. Astr. Soc., 102, 159, (1942).

2. C.R. Burch, "Application of the plate diagram to reflecting telescope design", Optica Acta, 26, No. 4, 493-504, (1979).

3. S.C.B. Gascoigne, "On Ritchey-Chretien systems", The Observatory, 85, 79, (1965).

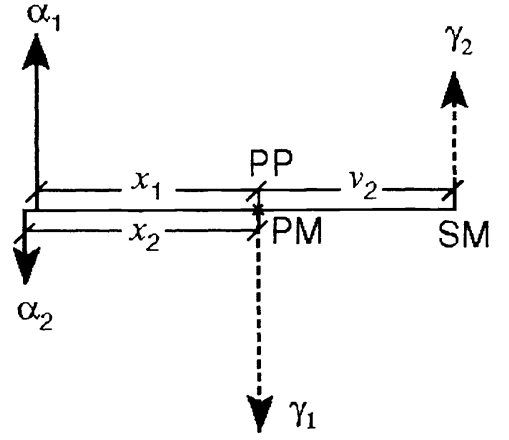

(a)

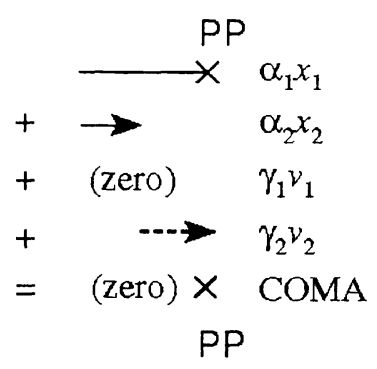

(b)

Figure 8. (a) See-saw diagram for a Ritchey-Chretien telescope and (b) schematic diagram for evaluation of coma. 